\title{
Flipping the switch: reverse-demand voltage-sensitive fluorophores
}

\author{
Jack T. McCann, ${ }^{\ddagger}$ Brittany R. Benlian, ${ }^{\S}$ Isaac J. Knudson, ${ }^{\ddagger}$ and Evan W. Miller ${ }^{\ddagger \S} *$ \\ Departments of ${ }^{\ddagger}$ Chemistry and ${ }^{\S}$ Molecular \& Cell Biology and ${ }^{\dagger}$ Helen Wills Neuroscience Institute. University of California, \\ Berkeley, California 94720, United States.
}

\begin{abstract}
Fluorescence microscopy with fluorescent reporters that respond to environmental cues are a powerful method for interrogating biochemistry and biophysics in living systems. Photoinduced electron transfer (PeT) is commonly used as a trigger to modulate fluorescence in response to changes in the biological environment. PeT based indicators rely either on PeT into the excited state (acceptor PeT) or out of the excited state (donor PeT). Our group has been developing voltage-sensitive fluorophores (VF dyes) that respond to changes in biological membrane potential. We hypothesize that the mechanism of voltage sensitivity arises from acceptor PeT (a-PeT) from an electron-rich aniline-containing molecular wire into the excited state fluorophore, resulting in decreased fluorescence at negative membrane potentials. Here, we can reverse the direction of electron flow to access donorexcited PeT (d-PeT) VF dyes by introducing electron-withdrawing (EWG), rather than electron-rich molecular wires. Similar to firstgeneration aniline containing VF dyes, EWG-containing VF dyes show voltage-sensitive fluorescence, but with the opposite polarity: hyperpolarizing membrane potentials now give fluorescence increases. We use a combination of computation and experiment to estimate a $\Delta E$ of $\sim 0.6 \mathrm{eV}$ for voltage sensitivity in d-PeT indicators, show that two of the new reverse VF dyes are voltage sensitive, and provide the first example, to our knowledge, of a molecular sensor that can be tuned across energy regimes to access bidirectional electron flow for fluorescence sensing in living systems.
\end{abstract}

Biological membrane potentials $\left(V_{m}\right)$ arise from differences in ion concentrations across a selectively-permeable lipid bilayer and are a defining feature of life. ${ }^{1}$ Despite the centrality of the maintenance and dynamics of $V_{m}$, methods to measure $V_{m}$ remain restricted primarily to electrode-based approaches like patch-clamp electrophysiology. Visualizing cellular $V_{m}$ with fluorescent indicators offers a powerful complement to traditional electrode methods and seeks to address problems of low-throughput, poor spatial resolution, and invasiveness associated with electrophysiology. ${ }^{2}$

Fluorescent dyes have long been used to monitor biologically relevant analytes, reactions, and properties. A number of innovative methods have emerged for toggling the properties of fluorescent molecules in response to changes in their environment. Among these, modulating photoinduced electron transfer (PeT) stands out as a powerful method for designing fluorescent reporters. ${ }^{3-6}$ By controlling PeT into or out of the excited state of a fluorophore based on the binding or reaction with a diverse array of analytes like $\mathrm{Ca}^{2+}, 7$ nitric oxide, ${ }^{8}{ }^{1} \mathrm{O}_{2},{ }^{9} \mathrm{Zn}^{2+},{ }^{10}$ or reactive oxygen species (ROS), ${ }^{11}$ PeT provides a generalizable trigger for designing fluorescent reporters.

Our group has been exploring the application of PeT-based triggers for monitoring $\mathrm{V}_{\mathrm{m}}{ }^{12}$ We postulate that voltage sensitivity within Voltage-sensitive Fluorophores (or VF dyes) arises from a $\mathrm{V}_{\mathrm{m}}$-sensitive electron transfer (Scheme 1); ${ }^{13-14}$ therefore, the direction of the electron transfer matters. If the electron moves from a molecular wire buried in the plasma membrane
Scheme 1. PeT in Voltage-sensitive Fluorophores (VF) dyes

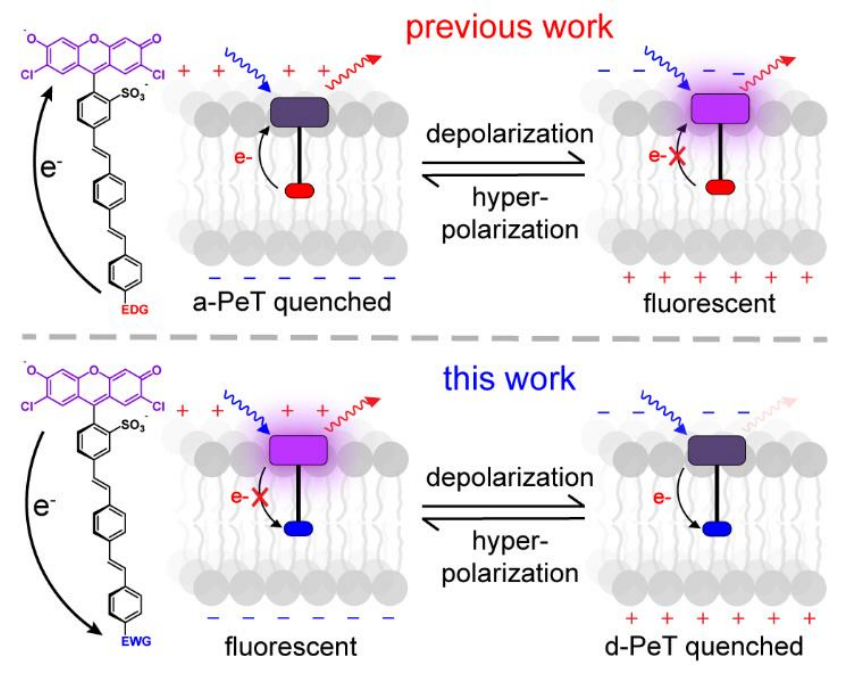

into a fluorophore on the extracellular face, PeT into the excited state fluorophore is fast at hyperpolarized potentials and the dye is $\operatorname{dim}$. At depolarized $V_{m}$, the voltage decreases the rate of PeT, allowing fluorescence to occur, and the dye brightens. Consistent with this hypothesis, VF dyes possess fluorescence turn-on responses upon membrane depolarization, ${ }^{12}$ nanosecond response kinetics, ${ }^{15}$ and voltagedependent fluorescence lifetimes. ${ }^{16}$ To date, all VF dyes make use of an aniline-containing molecular wire to achieve voltage- 

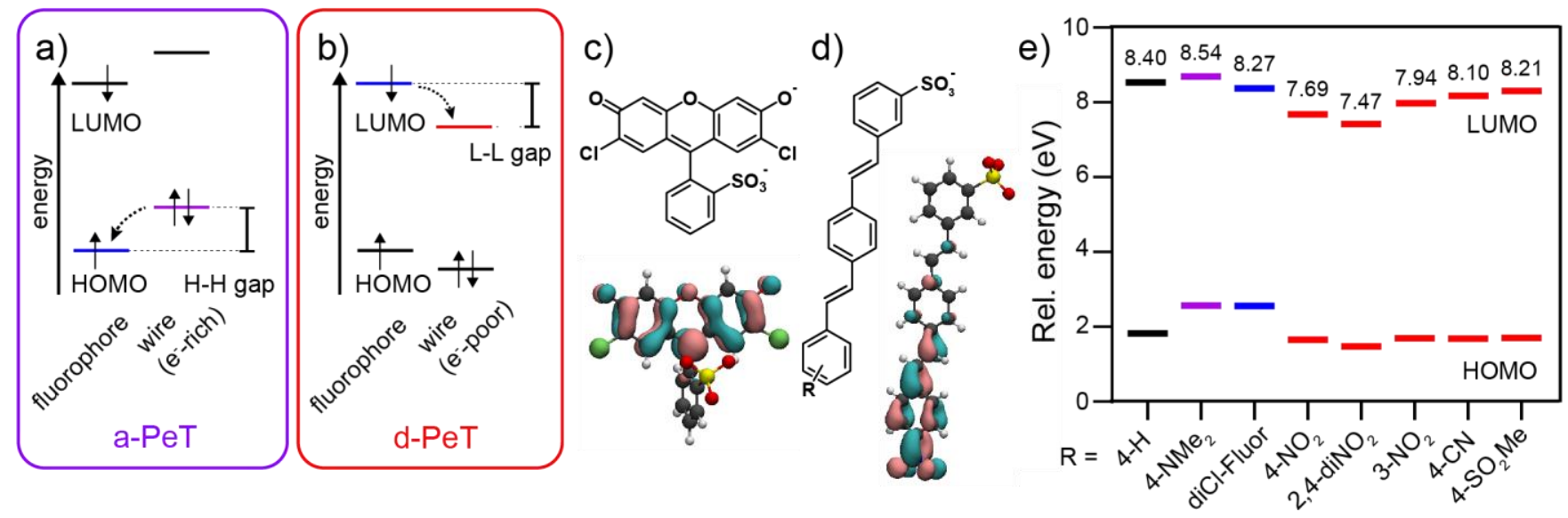

Figure 1. Controlling photoinduced electron transfer processes in voltage-sensitive fluorophores. Frontier molecular orbital diagrams for fluorescein and either a) electron-rich molecular wires that exhibit acceptor-excited PeT (a-PeT) or b) electron-poor molecular wires that exhibit donor-excited PeT (d-Pet). c) Structure and LUMO of 2',7'-dichloro-3-sulfonofluorescein. d) Structure and LUMO of electron poor molecular wires. This specific example is $4-\mathrm{NO}_{2} \mathrm{VF}$, but $\mathrm{R}$ can equal any of the substituents indicated in panel (e). e) Plot of calculated energy levels $(\mathrm{eV})$ of 2',7'-dichloro-3-sulfonofluorescein and various molecular wires with the indicated $\mathrm{R}$ group.

sensitivity in an acceptor-PeT (a-PeT) configuration in which the fluorophore acts as the electron acceptor.

However, if the hypothesis about the mechanism of voltage sensing is correct, replacing the electron-rich aniline with an electron-withdrawing group (EWG) should decrease the frontier molecular orbital energies of the wire and enable donor-excited PeT (d-PeT) (Scheme 1). In this configuration, hyperpolarized $\mathrm{V}_{\mathrm{m}}$ decrease the rate of PeT. This results in fluorescence brightening at hyperpolarized potentials and would provide the first example of a molecular sensor architecture with bi-directional electron flow for sensing in aPeT or d-PeT configurations.

Here we show that electron-poor molecular wires with EWG substituents can be incorporated into a VF dye scaffold, reversing the direction of electron flow, and inverting the sign of the fluorescence response to $\mathrm{V}_{\mathrm{m}}$ changes. We calculate the HOMO and LUMO energies of a series of EWG-containing molecular wires, synthesize 5 new EWG-VF dyes, characterize their spectroscopic properties, and evaluate their voltage sensitivity in mammalian cells. Two of the new dyes show voltage sensitivity, but with an inverted polarity relative to previously reported aniline-containing VF dyes. ${ }^{17-19}$

To investigate the possibility of reversing the polarity of VF dyes through d-PeT, we performed DFT calculations to estimate the relative HOMO and LUMO energies of the orthogonal fluorophore and molecular wire systems. We optimized geometry using def2-TZVP/WB97XD and calculated HOMO and LUMO energies for the fluorophore and molecular wire (Figure S1). ${ }^{20-21}$ Geometry optimization and DFT calculations on the complete 4- $\mathrm{NO}_{2}$ VF molecule (3a, Scheme 2) reveal that the HOMO is associated with the fluorophore, and the HOMO-1 is associated with the molecular wire. The LUMO is associated with the molecular wire, and the LUMO+1 is associated with the fluorophore (Figure S1). To save computational time, we split the entire 4- $\mathrm{NO}_{2}$ VF into two components, the fluorophore and the molecular wire (Figure
1), optimized geometries, and calculated HOMO and LUMO values (Figure 1). The HOMO and LUMO values calculated using the individual components gave values that matched well with those calculated using the entire VF dye (Figure S1). Therefore, we used calculations based on components, to save computational time-1440 cpu hours for the 66 atoms of the intact 4- $\mathrm{NO}_{2}$ VF compared with 270 and 288 cpu hours for the fluorophore and molecular wire, respectively-and to enable a rapid mix-and-match comparison of fluorophores and different molecular wires.

We find that for aniline-substituted VF2.1.Cl, ${ }^{17-19}$ the HOMO of the molecular wire is higher in energy than the HOMO of the fluorophore, with a HOMO-HOMO $(\mathrm{H}-\mathrm{H})$ gap of approximately $-0.05 \mathrm{eV}$, indicating the possibility of electron transfer from the wire to the fluorophore, or a-PeT. Conversely, the molecular wire LUMO of VF2.1.Cl is higher than the LUMO of $2^{\prime}, 7^{\prime}$ dichloro-3-sulfonofluorescein, with a LUMO-LUMO (L-L) gap of approximately $+0.27 \mathrm{eV}$, indicating that PeT from the fluorophore to the molecular wire (dPeT) is unlikely for this molecule (Figure 1). On the other hand, for EWG-VF dyes like 4- $\mathrm{NO}_{2} \mathrm{VF}$, we find the complementary configuration: the wire LUMO of $4-\mathrm{NO}_{2} \mathrm{VF}$ is lower than the fluorescein LUMO by approximately $-0.58 \mathrm{eV}$, indicating the possibility of PeT. ${ }^{22}$ The $\mathrm{HOMO}$ of the $4-\mathrm{NO}_{2}$ VF wire is $0.75 \mathrm{eV}$ lower than the HOMO of fluorescein, making a-PeT unlikely for this molecule (Figure 1). We calculated the orbital energies for other EWGcontaining molecular wires, 2,4-diNO $, 3-\mathrm{NO}_{2}, 4-\mathrm{CN}$, and 4$\mathrm{SO}_{2} \mathrm{Me}$ (Scheme 2, Figure 1). Both 4- $\mathrm{NO}_{2} \mathrm{VF}$ and 2,4-diNO $2 \mathrm{VF}$ possess L-L gaps of around $-0.6 \mathrm{eV}$ or larger. 3-NO $2 \mathrm{VF}$ had an intermediate $L-L$ gap value of $-0.33 \mathrm{eV}$, while the $\mathrm{L}-\mathrm{L}$ gaps for 4-CN VF and 4- $\mathrm{SO}_{2} \mathrm{Me}$ VF decrease substantially to -0.18 and $0.07 \mathrm{eV}$, respectively (Figure 1, Table 1). For comparison, the $\mathrm{L}-\mathrm{L}$ and $\mathrm{H}-\mathrm{H}$ gaps for VF2.0.Cl, which lacks an aniline or EWG substituent and is not voltage sensitive, are $+0.12 \mathrm{eV}$ and +0.61 $\mathrm{eV}$, respectively. 
Table 1. Properties of EWG-VFs

\begin{tabular}{|c|c|c|c|c|c|c|c|}
\hline Compound & $\mathrm{R}$ & $\Delta \mathrm{F} / \mathrm{F}^{\mathrm{a}}$ & $\mathrm{LUMO}(\mathrm{eV})^{\mathrm{b}}$ & $\mathrm{L}-\mathrm{L} \mathrm{Gap} \mathrm{(eV)})^{\mathrm{c}}$ & $\begin{array}{c}\lambda_{\mathrm{abs}^{\mathrm{d}}} \\
(\mathrm{nm})\end{array}$ & Rel. Cell Brightness & $\Phi_{\mathrm{fl}}{ }^{\mathrm{f}}$ \\
\hline $3 \mathrm{a}$ & $4-\mathrm{NO}_{2}$ & $-3.8 \pm 0.1$ & 7.69 & -0.58 & 387 & 2.2 & 0.23 \\
\hline $3 \mathrm{~b}$ & $2,4-\mathrm{diNO}_{2}$ & $-8.2 \pm 0.7$ & 7.45 & -0.79 & 397 & 0.15 & 0.10 \\
\hline $3 \mathrm{c}$ & $3-\mathrm{NO}_{2}$ & $-0.4 \pm 0.01$ & 7.94 & -0.33 & 342 & 1.2 & 0.71 \\
\hline $3 \mathrm{~d}$ & $4-\mathrm{CN}$ & $-0.4 \pm 0.1$ & 8.09 & -0.18 & 366 & 0.99 & 0.71 \\
\hline $3 \mathrm{e}$ & $4-\mathrm{SO}_{2} \mathrm{Me}$ & $0 \pm 0.7$ & 8.21 & -0.07 & 360 & 0.10 & 0.51 \\
\hline $\mathrm{VF2.1.Cl}$ & $4-\mathrm{NMe}_{2}$ & $25 \pm 1$ & 8.54 & +0.27 & $389 \mathrm{~g}$ & 1.0 & $0.12^{\mathrm{g}}$ \\
\hline $\mathrm{VF2} 2.0 . \mathrm{Cl}$ & $\mathrm{H}$ & 0 & 8.40 & +0.12 & $360 \mathrm{~g}$ & --- & $0.83^{\mathrm{g}}$ \\
\hline
\end{tabular}

a per $100 \mathrm{mV}$ in HEK293T cells. ${ }^{b}$ Values normalized to respective sulfonate orbital. ${ }^{c}$ Difference between LUMO of dichlorosulfononfluorescein and corresponding molecular wire. ${ }^{d}$ For molecular wire; acquired in $0.1 \mathrm{M} \mathrm{EtOH-KOH}$. e Determined in HEK293T cells; all values relative to brightness of VF2.1.Cl. ${ }^{\mathrm{f}}$ Determined in $\mathrm{EtOH}-\mathrm{KOH} .{ }^{\mathrm{g}}$ Values from Boggess, et al. RSC Chem Biol, 2021, 2, 248-58. ${ }^{19}$

Scheme 2. Synthesis of EWG-containing VF dyes

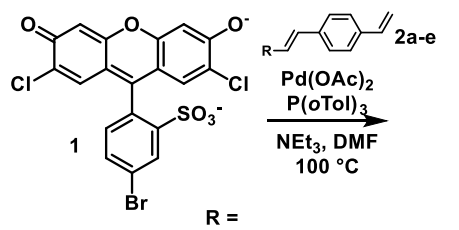

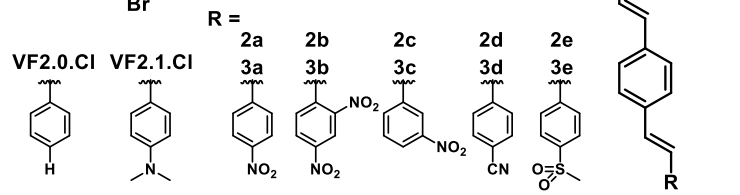

Based on these data, we hypothesize that strongly EWG substitution, like 4- $\mathrm{NO}_{2}$ and 2,4-diNO $\mathrm{N}_{2}$, might enable d-PeT VF dyes because of their large, negative L-L gaps.

To test the hypothesis that VF dyes could be "run in reverse", we synthesized 5 different EWG-substituted stilbene derivatives. 4- $\mathrm{NO}_{2}, 3-\mathrm{NO}_{2}$, and 4-CN stilbenes could all be accessed via sequential Wittig/Heck/Wittig reactions, with the Wittig reactions carried out with $\mathrm{K}_{2} \mathrm{CO}_{3}$ as the base (Scheme S1). More electron-withdrawing 2,4-diNO 2 stilbene required a different route, since use of $\mathrm{K}_{2} \mathrm{CO}_{3}$ in the initial Wittig olefination caused decomposition. Instead, we synthesized 2,4-diNO $\mathrm{N}_{2}$ and $4-\mathrm{SO}_{2} \mathrm{Me}$ stilbene $(\mathbf{2 b}, \mathbf{2 e})$ via a Horner Wadsworth-Emmons reaction (Scheme S2). Pd-catalyzed cross coupling of the EWG-substituted stilbenes (2a-e) with 5bromo-2',7'-dichloro-3-sulfono-fluorescein (1) afforded EWGVF dyes (3a-e).

All of the EWG-VF dyes display an invariant absorption maximum at $520 \mathrm{~nm}$ and emission maximum at $540 \mathrm{~nm}$, owing to the common 2',7'-dichloro-3-sulfonofluorescein fluorophore (Figure 2). The absorbance band corresponding to the molecular wire is different for each of the new EWG-VF dyes: 3-NO 2 VF is shortest at $366 \mathrm{~nm}$, followed by 4-CN VF and 4-SO ${ }_{2} \mathrm{Me} \mathrm{VF}$ at $375 \mathrm{~nm}, 4-\mathrm{NO}_{2} \mathrm{VF}$ at $387 \mathrm{~nm}$, and 2,4-diNO 2 VF at $397 \mathrm{~nm}$ (Figure 2). For comparison, the molecular wire absorbance band of VF2.1.Cl is approximately $390 \mathrm{~nm}$. The quantum yield of fluorescence $\left(\Phi_{\mathrm{fl}}\right)$ for EWG-VF dyes ranges from 0.09 to 0.71 (Table 1). Both $4-\mathrm{NO}_{2} \mathrm{VF}$ and $2,4-\mathrm{diNO}_{2} \mathrm{VF}$ have relatively low $\Phi_{\mathrm{fl}}$ values, indicating a high degree of PeT quenching.

All of the EWG-VF dyes stain the plasma membranes of

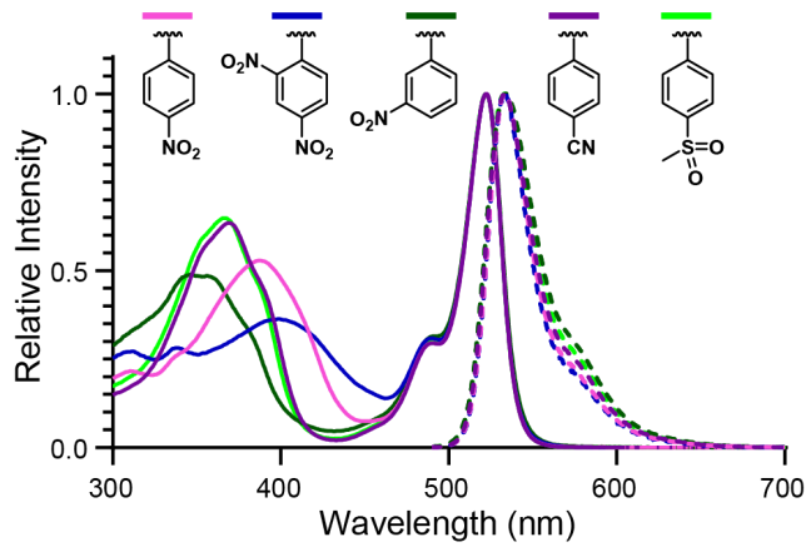

Figure 2. UV-vis and emission spectra of reverse VF dyes. Plot of relative intensity vs wavelength for indicated reverse VF dyes. Absorbance is shown in solid lines; emission is shown in dashed lines. Each absorbance spectrum is normalized to the $\lambda_{\max }$; each emission spectrum is normalized to the $\lambda_{\max }$. Spectra were acquired with $1.25 \mathrm{uM}$ dye in $0.1 \mathrm{M} \mathrm{KOH}$ in EtOH buffer. For emission spectra, excitation was provided at $480 \mathrm{~nm}$.

HEK293T cells (Figure 3). Widefield epifluorescence microscopy reveals a "chicken-wire" pattern of cellular staining, indicating exclusion of the dyes from the cytosol and intercalation into the plasma membrane. When compared to aniline-substituted VF2.1. Cl, 4- $\mathrm{NO}_{2}$ VF has the brightest membrane staining, 2-fold brighter than VF2.1.Cl (Figure 3, Table 1). 3- $\mathrm{NO}_{2}$ VF and 4-CN VF both have similar brightness compared to VF2.1. Cl, and 2,4-diNO $\mathrm{N}_{2} \mathrm{VF}$ and 4- $\mathrm{SO}_{2} \mathrm{Me} \mathrm{VF}$ have the lowest cellular fluorescence, approximately 10 -fold less bright than VF2.1.Cl (Figure 3, Table 1).

Finally, we assessed the voltage sensitivity of the new, EWGVF dyes in HEK293T cells using whole-cell patch clamp electrophysiology. EWG-VF dyes with nitro substituents show voltage sensitivity, but with a reverse polarity (Figure 4). Unlike aniline-substituted VF2.1.Cl, which displays a fluorescence increase upon membrane depolarization, the fluorescence of 4- $\mathrm{NO}_{2}$ VF and 2,4-diNO 2 VF decreases upon depolarization and becomes brighter upon hyperpolarization.

The other EWG-VFs, 4-CN VF, 4- $\mathrm{SO}_{2} \mathrm{Me} \mathrm{VF}$, and 3- $\mathrm{NO}_{2} \mathrm{VF}$ show no voltage sensitivity. In agreement with our hypothesis, 

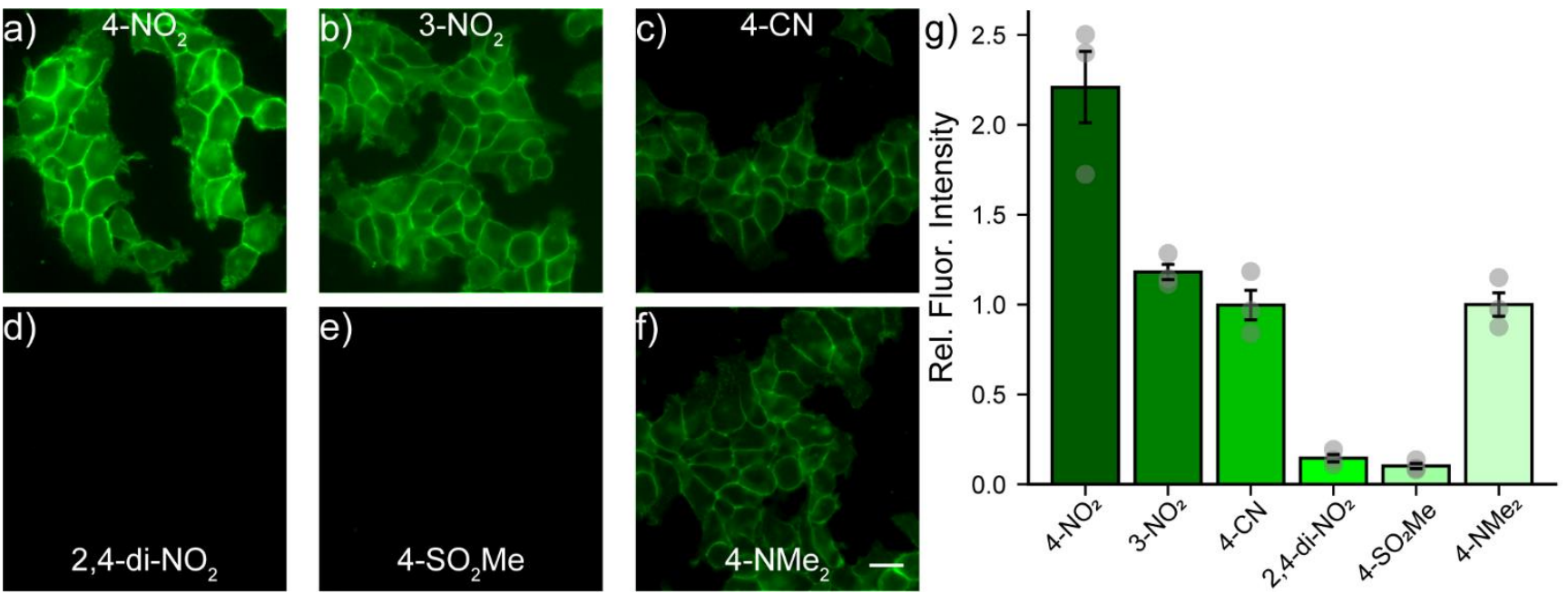

Figure 3. Live cell imaging with reverse VF dyes. Widefield epifluorescence images of HEK293T cells treated with either a) 4-NO $2 \mathrm{VF}(6)$, b) 3- $\mathrm{NO}_{2} \mathrm{VF}(\mathbf{1 7})$, c) 4-CN VF (27), d) 2,4-diNO 2 VF (13), e) 4-SO $\mathrm{SO}_{2} \mathrm{VF}(\mathbf{2 3})$, or f) VF2.1.Cl. All dyes were loaded at $250 \mathrm{nM}$. Scale bar is $20 \mu \mathrm{m}$. g) Plot of cellular fluorescence intensity of HEK293T cells loaded with the indicated dye. Data are mean \pm S.E.M. for $n=3$ independent experiments. For each experiment, or coverslip, we analyzed between $50-200$ cells and took the mean fluorescence intensity.
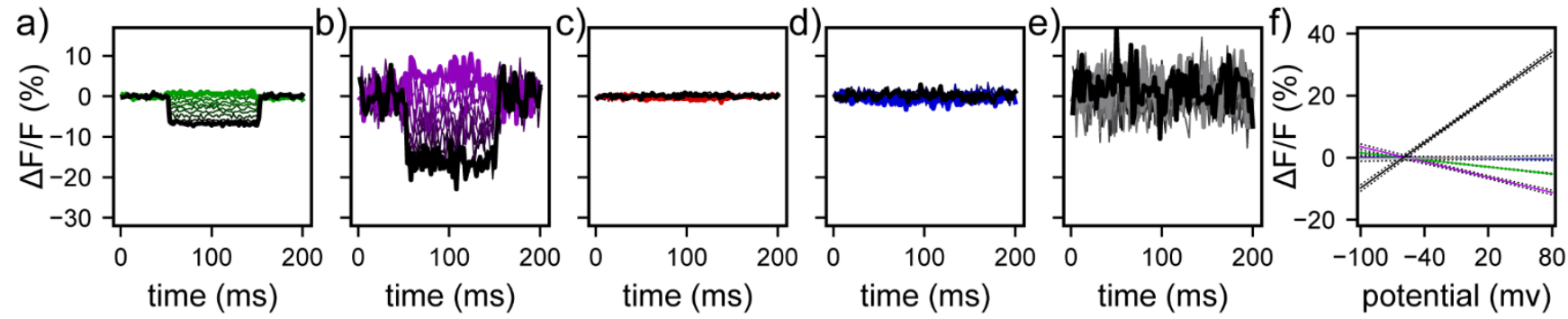

Figure 4. Voltage sensitivity of $V F$ dyes. Plots of $\Delta F / F$ vs time for HEK293T cells loaded with either a) 4- $\mathrm{NO}_{2} \mathrm{VF}$, b) $\left.2,4-\mathrm{diNO} \mathrm{V}_{2} \mathrm{VF}, \mathrm{c}\right)$ 3-NO $\mathrm{VF}$, d) 4-CN VF, or e) 4- $\mathrm{SO}_{2} \mathrm{Me} V \mathrm{~F}$. Cells were held at $-60 \mathrm{mV}$ under whole-cell voltage-clamp conditions and then stepped to potentials ranging from $+100 \mathrm{mV}$ to $-100 \mathrm{mV}$ in $20 \mathrm{mV}$ increments. f) Plot of $\Delta \mathrm{F} / \mathrm{F}$ per $100 \mathrm{mV}$ vs membrane potential (in $\mathrm{mV})$ for 4-NO $2 \mathrm{VF}(\mathrm{green}, \mathrm{n}=$ 8), 2,4-diNO 2 VF (magenta, $n=5$ ), 3- $\mathrm{NO}_{2}$ VF (red, $n=5$ ), 4-CN VF (blue, $n=3$ ), 4-SO $\mathrm{SMe} \mathrm{VF}_{2}$ (gray, $n=4$ ), or VF2.1.Cl (black). Data are mean \pm S.E.M. VF2.1.Cl data is from Turnbull, et al. J Am Chem Soc 2021, 143 (16), 6194-6201.23

4- $\mathrm{NO}_{2}$ VF and 2,4-diNO ${ }_{2}$ VF have the largest $\mathrm{L}-\mathrm{L}$ gaps, corresponding to higher values of $\Delta \mathrm{G}_{\mathrm{PeT}}$. A LUMO-LUMO gap of approximately $0.6 \mathrm{eV}$ is required for voltage sensitivity in EWGVF dyes, consistent with previous reports. ${ }^{22} 4-\mathrm{NO}_{2}$ VF has an approximate $-4 \% \Delta \mathrm{F} / \mathrm{F}$ per $100 \mathrm{mV}$ in HEK293T cells, while 2,4diNO $_{2}$ VF has about an $-8 \%$ voltage sensitivity (Figure 4, Table 1). However, despite the higher nominal voltage sensitivity, 2,4-diNO $\mathrm{VF}_{2}$ shows very low cellular fluorescence (Figure S2), approximately 40 -folder lower than $4-\mathrm{NO}_{2} \mathrm{VF}$, making 4- $\mathrm{NO}_{2}$ VF the most useful EWG-VF for cellular voltage imaging.

In summary, we report the design, synthesis, and validation of a new class of $d$-PeT based VFs for voltage imaging. DFT was used to determine the orbital energies of different molecular wires with EWGs and the 2',7'-dichloro-3-sulfonofluorescein fluorophore. This approach allowed us to mix-and-match LUMO energies by normalizing to a shared orbital (Figure S3), leading to much lower computational cost compared to calculations on full VF molecules. Five d-PeT VF targets were synthesized to test the validity of our computational method (Scheme 1). Both 4- $\mathrm{NO}_{2} \mathrm{VF}$ and 2,4-diNO $\mathrm{NF}_{2} \mathrm{VF}$ are voltage sensitive, but with a response polarity inverted compared to aniline-containing VF indicators. While a-PeT and d-PeT strategies have been widely used to design fluorescent reporters, ${ }^{5}$ this is the first demonstration that HOMO/LUMO levels can be tuned across ranges that give access to both aPeT and d-PeT for detection of a biologically-relevant analyte with the same fluorophore scaffold. Future directions include improving voltage sensitivity by pairing electron-deficient molecular wires with electron-rich fluorophores and applying reverse VF indicators to biological contexts where the membrane potential polarity is switched compared cellular membranes, for example in lipid bilayer-enclosed organelles. ${ }^{24-}$ 25

\section{ASSOCIATED CONTENT}

Supporting Information. Experimental procedures, NMR spectra, and supporting figures. This material is available free of charge via the Internet.

\section{AUTHOR INFORMATION}

\section{Corresponding Author}


* evanwmiller@berkeley.edu

\section{ACKNOWLEDGMENT}

E.W.M. acknowledges support from the Camille Dreyfus Foundation and NIH (R35GM119855). J.T.M., B.R.B., and I.J.K. were supported, in part, by a training grant from $\mathrm{NIH}$ (T32GM066698). We thank Dr. Hasan Celik and the staff of the College of Chemistry NMR facility for their assistance. Instruments in CoC-NMR are supported in part by NIH S10OD024998. 900MHz NMR supported by NIH GM68933. We thank Drs. Kathleen Durkin and Dave Small for their assistance with computations. The CoCMGCF is supported by NIH S10OD023532.

\section{REFERENCES}

1. Kandel, E. R.; Schwartz, J. H.; Jessell, T. M.; Siegelbaum, S. A.; Hudspeth, A. J.; Mack, S., Principles of neural science. 5th ed. ed.; McGraw-Hill Education LLC: New York, N.Y, 2013.

2. Peterka, D. S.; Takahashi, H.; Yuste, R., Imaging voltage in neurons. Neuron 2011, 69 (1), 9-21.

3. de Silva, P. A.; Gunaratne, N. H. Q.; McCoy, C. P., A molecular photoionic AND gate based on fluorescent signalling. Nature 1993, 364 (6432), 42-44.

4. Daly, B.; Ling, J.; de Silva, A. P., Current developments in fluorescent PET (photoinduced electron transfer) sensors and switches. Chemical Society Reviews 2015, 44 (13), 4203-4211.

5. Nagano, T., Development of fluorescent probes for bioimaging applications. Proc Jpn Acad Ser B Phys Biol Sci 2010, 86 (8), 837-847.

6. Lavis, L. D., Teaching Old Dyes New Tricks: Biological Probes Built from Fluoresceins and Rhodamines. Annual Review of Biochemistry 2017, 86 (1), 825-843.

7. Minta, A.; Kao, J. P. Y.; Tsien, R. Y., Fluorescent indicators for cytosolic calcium based on rhodamine and fluorescein chromophores. Journal of Biological Chemistry 1989, 264 (14), 81718178.

8. Kojima, H.; Nakatsubo, N.; Kikuchi, K.; Kawahara, S.; Kirino, Y.; Nagoshi, H.; Hirata, Y.; Nagano, T., Detection and Imaging of Nitric Oxide with Novel Fluorescent Indicators: Diaminofluoresceins. Analytical Chemistry 1998, 70 (13), 2446-2453.

9. Tanaka, K.; Miura, T.; Umezawa, N.; Urano, Y.; Kikuchi, K.; Higuchi, T.; Nagano, T., Rational Design of Fluorescein-Based Fluorescence Probes. Mechanism-Based Design of a Maximum Fluorescence Probe for Singlet Oxygen. Journal of the American Chemical Society 2001, 123 (11), 2530-2536.

10. Burdette, S. C.; Walkup, G. K.; Spingler, B.; Tsien, R. Y.; Lippard, S. J., Fluorescent Sensors for Zn2+ Based on a Fluorescein Platform: Synthesis, Properties and Intracellular Distribution. Journal of the American Chemical Society 2001, 123 (32), 7831-7841.

11. Ueno, T.; Urano, Y.; Setsukinai, K.-i.; Takakusa, H.; Kojima, H.; Kikuchi, K.; Ohkubo, K.; Fukuzumi, S.; Nagano, T., Rational Principles for Modulating Fluorescence Properties of Fluorescein. Journal of the American Chemical Society 2004, 126 (43), 1407914085.
12. Liu, P.; Miller, E. W., Electrophysiology, Unplugged: Imaging Membrane Potential with Fluorescent Indicators. Accounts of Chemical Research 2020, 53 (1), 11-19.

13. de Silva, A. P.; Gunaratne, H. Q. N.; Habib-Jiwan, J.-L.; McCoy, C. P.; Rice, T. E.; Soumillion, J.-P., New Fluorescent Model Compounds for the Study of Photoinduced Electron Transfer: The Influence of a Molecular Electric Field in the Excited State. Angewandte Chemie International Edition in English 1995, 34 (16), 1728-1731.

14. Li, L.-S., Fluorescence Probes for Membrane Potentials Based on Mesoscopic Electron Transfer. Nano Letters 2007, 7 (10), 2981-2986.

15. Beier, H. T.; Roth, C. C.; Bixler, J. N.; Sedelnikova, A. V.; Ibey, B. L., Visualization of Dynamic Sub-microsecond Changes in Membrane Potential. Biophysical Journal 2019, 116 (1), 120-126.

16. Lazzari-Dean, J. R.; Gest, A. M. M.; Miller, E. W., Optical estimation of absolute membrane potential using fluorescence lifetime imaging. elife 2019, 8, e44522.

17. Woodford, C. R.; Frady, E. P.; Smith, R. S.; Morey, B.; Canzi, G.; Palida, S. F.; Araneda, R. C.; Kristan, W. B.; Kubiak, C. P.; Miller, E. W.; Tsien, R. Y., Improved PeT Molecules for Optically Sensing Voltage in Neurons. Journal of the American Chemical Society 2015, 137 (5), 1817-1824.

18. Miller, E. W.; Lin, J. Y.; Frady, E. P.; Steinbach, P. A.; Kristan, W. B.; Tsien, R. Y., Optically monitoring voltage in neurons by photoinduced electron transfer through molecular wires. Proceedings of the National Academy of Sciences 2012, 109 (6), 2114-2119.

19. Boggess, S. C.; Lazzari-Dean, J. R.; Raliski, B. K.; Mun, D. M.; Li, A. Y.; Turnbull, J. L.; Miller, E. W., Fluorescence lifetime predicts performance of voltage sensitive fluorophores in cardiomyocytes and neurons. RSC Chemical Biology 2021, 2 (1), 248-258.

20. Chai, J.-D.; Head-Gordon, M., Long-range corrected hybrid density functionals with damped atom-atom dispersion corrections. Physical Chemistry Chemical Physics 2008, 10 (44), 6615-6620.

21. Weigend, F.; Ahlrichs, R., Balanced basis sets of split valence, triple zeta valence and quadruple zeta valence quality for $\mathrm{H}$ to Rn: Design and assessment of accuracy. Physical Chemistry Chemical Physics 2005, 7 (18), 3297-3305.

22. Chi, W.; Chen, J.; Liu, W.; Wang, C.; Qi, Q.; Qiao, Q.; Tan, T. M.; Xiong, K.; Liu, X.; Kang, K.; Chang, Y.-T.; Xu, Z.; Liu, X., A General Descriptor $\Delta E$ Enables the Quantitative Development of Luminescent Materials Based on Photoinduced Electron Transfer. Journal of the American Chemical Society 2020, 142 (14), 6777-6785.

23. Turnbull, J. L.; Benlian, B. R.; Golden, R. P.; Miller, E. W., Phosphonofluoresceins: Synthesis, Spectroscopy, and Applications. Journal of the American Chemical Society 2021, 143 (16), 6194-6201.

24. Klier, P. E. Z.; Martin, J. G.; Miller, E. W., Imaging Reversible Mitochondrial Membrane Potential Dynamics with a Masked Rhodamine Voltage Reporter. J Am Chem Soc 2021, 143 (11), 40954099.

25. Saminathan, A.; Devany, J.; Veetil, A. T.; Suresh, B.; Pillai, K. S.; Schwake, M.; Krishnan, Y., A DNA-based voltmeter for organelles. Nature Nanotechnology 2021, 16 (1), 96-103.

Reverse-demand voltage-sensitive fluorophores

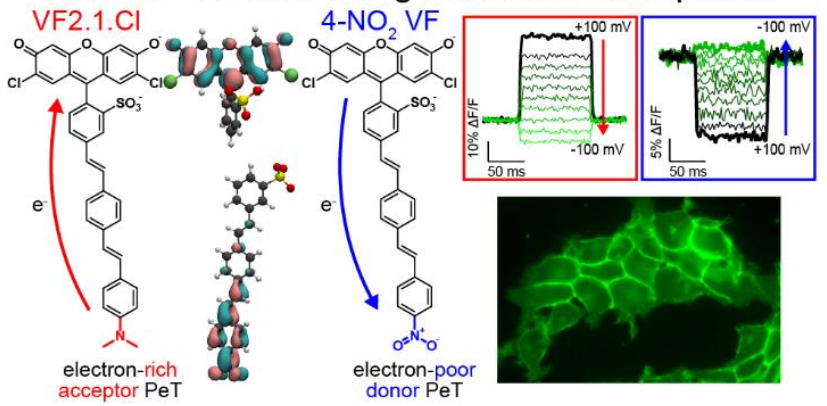

